\title{
INFLUÊNCIA DA QUALIDADE DOS CONTEXTOS FAMILIAR E DE CRECHE NO ENVOLVIMENTO E NO DESENVOLVIMENTO DA CRIANÇA
}

\author{
Manuela Pessanha \\ Ana Isabel Pinto ${ }^{2}$ \\ Silvia Barros ${ }^{3}$
}

Resumo: O presente estudo analisou a influência da qualidade dos contextos educativos (casa e creche) no envolvimento da criança com adultos, pares e objectos, observado em contexto de creche, e no seu desenvolvimento cognitivo, social e de linguagem. Participaram 120 crianças do Distrito do Porto, respectivas famílias e educadores. Os resultados indicaram que características da criança, a qualidade da sala de creche e os comportamentos interactivos dos educadores influenciam o envolvimento da criança em diferentes níveis de sofisticação. Verificou-se a existência de efeitos de pequena magnitude da qualidade das salas de creche nos resultados desenvolvimentais. Evidenciou-se também a existência de efeitos, pequenos a moderados, da qualidade do ambiente familiar no desenvolvimento das crianças.

Palavras-chave: envolvimento, desenvolvimento, qualidade dos contextos, interacções

Effects of Day Care and Home Quality on Children's Engagement and Development (Abstract): This study examined the effects of day care and home quality both on children's observed engagement with adults, peers, and objects, in day care classrooms, and on their cognitive, social and linguistic developmental outcomes. One hundred and twenty toddlers from Porto district, their families and teachers participated in the study. Results showed that child characteristics, daycare quality, and teachers' interactive behaviours influenced children's engagement at different sophistication levels. Small effects of daycare quality on chil-

\footnotetext{
${ }^{1}$ Faculdade de Psicologia e de Ciências da Educação, Universidade do Porto

2 Faculdade de Psicologia e de Ciências da Educação, Universidade do Porto

${ }^{3}$ Faculdade de Psicologia e de Ciências da Educação, Universidade do Porto

Este trabalho foi realizado no âmbito do projecto A qualidade das interacções em contexto familiar e de creche e a sua influência no desenvolvimento sociocognitivo da criança, coordenado pelo Professor Joaquim Bairrão no Centro de Psicologia - Universidade do Porto (2000-2004) e financiado pela FCT (POCTI/PSI/35207/2000).
}

PSICOLOGIA, Vol. XXIII (2), 2009, Edições Colibri, Lisboa, pp. 55-71 
dren's developmental outcomes were found. Results indicated small to moderate effects of home quality on children's developmental outcomes.

Key Words: engagement, development, environmental quality, interactions

\section{Introdução}

Este projecto pretendeu estudar o envolvimento enquanto variável socio-cognitiva, de acordo com a hierarquia de base desenvolvimental conceptualizada por McWilliam e Bailey (1995), em crianças com idades compreendidas entre os 12 e os 36 meses.

A natureza transaccional do desenvolvimento (Sameroff \& Fiese, 2000) implica assumir que este resulta da interacção dinâmica e contínua entre factores biológicos e ambientais. Esta perspectiva implica uma abordagem ecológica (e.g., Bronfenbrenner \& Morris, 1998), que integra as características em evolução da pessoa em interacção com o ambiente e que considera diversos níveis de análise, desde factores de carácter biológico a características mais abrangentes da ecologia do desenvolvimento humano.

O estudo do envolvimento da criança durante os primeiros anos de vida é especialmente relevante, na medida em que se assume que descreve o processo através do qual as crianças adquirem conhecimento acerca do mundo. Considerando que as crianças em idade de creche e pré-escolar aprendem essencialmente através do jogo e de outras interacções com o seu meio ambiente, o grau em que elas se envolvem durante essas interacções deverá constituir um factor crítico para que ocorram níveis óptimos de aprendizagem (McWilliam \& Bailey, 1995). No presente estudo, para descrever as competências da criança observámos as suas interacções nas salas de creche, no decurso de experiências de vida diária diárias. McWilliam e Bailey (1995, p. 124) definiram envolvimento como a quantidade de tempo que a criança despende a interagir activa ou atentamente com o seu ambiente (com adultos, pares ou materiais) de uma forma desenvolvimental e contextualmente adequada em diferentes níveis de competência. $\mathrm{O}$ critério de adequação desenvolvimental requer que o comportamento seja apropriado à idade desenvolvimental e às capacidades da criança e o de adequação contextual requer que o comportamento seja adequado à actividade desenvolvida e às expectativas da situação. Estes critérios estiveram na base da definição dos diferentes níveis de envolvimento, organizados numa hierarquia de carácter desenvolvimental, com os comportamentos a variarem de um nível inferior para um nível mais sofisticado (McWilliam \& de Kruif, 1998). Os autores 
definiram nove niveis de envolvimento (i.e., persistente, simbólico, codificado, construtivo, diferenciado, atenção focalizada, indiferenciado, atenção ocasional e não envolvido) e quatro tipos de envolvimento (i.e., pares, adultos, objectos e self) (Quadro 1). Os tipos de envolvimento estão organizados numa hierarquia em que o envolvimento com pares tem prioridade sobre os outros tipos e o envolvimento com pares ou adultos tem prioridade sobre o envolvimento com objectos ou o self.

$\mathrm{O}$ envolvimento de qualidade superior constitui um factor mediador potencialmente crítico na aprendizagem e no desenvolvimento da criança desde os primeiros anos de vida (e.g., McWilliam \& Bailey, 1995). Os níveis de envolvimento em contexto de creche variam em função de características da criança (e.g., estatuto desenvolvimental e temperamento) e de aspectos do ambiente educativo, nomeadamente o ambiente físico e social e as características do educador (Raspa, McWilliam, \& Ridley, 2001). À medida que as idades cronológica e desenvolvimental das crianças aumentam, o nível de sofisticação do seu envolvimento também aumenta (de Kruif \& McWilliam, 1999; Pinto, Barros, Aguiar, Pessanha, \& Bairrão, 2006).

Tem sido igualmente documentada a importância da qualidade dos contextos de educação de infância para o desenvolvimento da criança, tanto a nível de dimensões de estrutura (e.g., rácio adulto:criança) como de dimensões de processo (e.g., ambientes seguros e estimulantes, interacções calorosas e responsivas) (NICHD ECCRN, 2006; Shonkoff \& Phillips, 2000).

Os efeitos da participação das crianças em contextos extrafamiliares de prestação de cuidados de elevada qualidade, em idades precoces, aparece ainda associada, de forma positiva, a curto e a longo prazo, aos seus resultados desenvolvimentais, principalmente nas que são oriundas de ambientes mais desfavorecidos ou menos estimulantes (e.g., Campbell, Wasik, Pungello, Burchinal, Barbarin, Kainz, K. et al., 2008).). Poucos estudos têm clarificado as relações entre aspectos da qualidade dos ambientes educativos $\mathrm{e}$ comportamentos de envolvimento das crianças em idades precoces. Neste contexto, o objectivo principal deste estudo foi o de examinar a influência da qualidade dos contextos de socialização, família e creche, no desenvolvimento cognitivo, social e da linguagem, bem como nos níveis de envolvimento da criança em contexto de creche. 
Quadro 1: Definições dos níveis de envolvimento observado

\begin{tabular}{|c|c|}
\hline Níveis de Envolvimento & Definições \\
\hline Persistência(a) & $\begin{array}{l}\text { Implica algum tipo de resolução de problemas e algum desafio; inclui } \\
\text { mudar de estratégia ou utilizar a mesma estratégia; requer comporta- } \\
\text { mentos dirigidos a um objectivo. } \\
\text { Exemplo: uma criança tenta completar um puzzle mas tem dificuldade } \\
\text { em ajustar as peças e tenta diversas formas de as encaixar. }\end{array}$ \\
\hline Simbólico (a) & $\begin{array}{l}\text { Implica o uso de formas convencionais de comportamento, como, a } \\
\text { linguagem, o jogo de faz-de-conta, linguagem simbólica desenhos, } \\
\text { etc. Requer descontextualização. } \\
\text { Exemplo: uma criança numa área de jogo dramático está a fazer de } \\
\text { conta que faz o jantar. }\end{array}$ \\
\hline Codificado (a) & $\begin{array}{l}\text { Implica a utilização de formas convencionais de comportamento que } \\
\text { estão relacionadas com o contexto e que dependem de referentes. } \\
\text { Inclui linguagem compreensível. } \\
\text { Exemplo: A criança está a falar com o educador acerca do jogo que } \\
\text { ambos estão a jogar. }\end{array}$ \\
\hline Construtivo (a) & $\begin{array}{l}\text { Implica manipulação de objectos para criar ou construir alguma } \\
\text { coisa. Inclui juntar objectos em qualquer tipo de forma espacial. } \\
\text { Requer intencionalidade. } \\
\text { Exemplo: A criança está a desenhar ou a construir uma torre. }\end{array}$ \\
\hline Diferenciado & $\begin{array}{l}\text { Implica coordenação e regulação do comportamento reflectindo } \\
\text { elaboração e progresso em direcção a formas convencionais de com- } \\
\text { portamento. Inclui interacção activa com o meio. } \\
\text { Exemplo: A criança utiliza uma colher para comer durante a refei- } \\
\text { ção. }\end{array}$ \\
\hline $\begin{array}{c}\text { Atenção } \\
\text { Focalizada }\end{array}$ & $\begin{array}{l}\text { Implica observar ou escutar estímulos do meio durante pelo menos } 3 \\
\text { segundos. Inclui características físicas da criança como: a expressão } \\
\text { facial séria e o acalmar da actividade motora. } \\
\text { Exemplo: A criança olha para o educador enquanto ele lê um livro } \\
\text { numa roda para o grande grupo. }\end{array}$ \\
\hline Indiferenciado (b) & $\begin{array}{l}\text { Implica observar ou escutar estímulos do meio durante pelo menos } 3 \\
\text { segundos. Inclui características físicas da criança como: a expressão } \\
\text { facial séria e o acalmar da actividade motora. } \\
\text { Exemplo: A criança olha para o educador enquanto ele lê um livro } \\
\text { numa roda para o grande grupo. }\end{array}$ \\
\hline $\begin{array}{c}\text { Atenção } \\
\text { Ocasional (b) }\end{array}$ & $\begin{array}{l}\text { Implica atenção descontraída e vaga; inclui monitorização do } \\
\text { ambiente por parte da criança. } \\
\text { Exemplo: Criança olha à volta da sala procurando o educador. }\end{array}$ \\
\hline $\begin{array}{c}\text { Não } \\
\text { Envolvido }\end{array}$ & $\begin{array}{l}\text { Implica falta de ocupação; requer a ausência de todos os outros } \\
\text { comportamentos. } \\
\text { Exemplo: uma criança empurra outra criança enquanto está numa } \\
\text { fila; uma criança fica sentada a olhar no vazio durante o horário de } \\
\text { actividades. }\end{array}$ \\
\hline
\end{tabular}

Nota: Envolvimento Sofisticado; (b) Envolvimento Não Sofisticado Adaptado de: Raspa, McWilliam, \& Ridley, 2001. 


\section{Método}

\section{Participantes}

Participaram neste estudo 120 crianças com idades entre 14 e 49 meses $(M=26, D P=7.07)$ que frequentavam 30 salas de creche no Distrito do Porto e respectivas famílias, em que o nível educacional médio das mães era de 10.83 anos $(D P=4.47)$. As salas (15 para $1-2$ anos e 15 para 2-3 anos), integradas em 15 creches, foram seleccionadas aleatoriamente, com uma taxa de participação de 58\%. Cinco salas eram da responsabilidade de auxiliares de acção educativa e as restantes da responsabilidade de educadoras de infância, todas do sexo feminino. Cerca de $84 \%$ dos responsáveis ${ }^{4}$ pelas salas possuía formação de grau superior e 33\% possuía cinco ou menos anos de experiência. Seleccionaram-se aleatoriamente 8 crianças de cada creche (4 da sala dos 1-2 anos e 4 da sala dos 2-3 anos, 2 do sexo masculino e 2 do feminino em cada sala). A taxa de participação das crianças e famílias foi de $71 \%$.

\section{Medidas e Recolha de dados}

Envolvimento Observado. Foi utilizado o Sistema de Avaliação da Qualidade do Envolvimento III (SAQE III) traduzido do Engagement Quality Observation System III (E-QUAL III; McWilliam \& de Kruif, 1998), um procedimento por amostras no tempo, que engloba uma dimensão quantitativa, relativa à proporção de tempo de envolvimento, e uma dimensão qualitativa, relativa ao nível e tipo de envolvimento. Os autores definiram nove níveis e quatro tipos de envolvimento, exaustivos e mutuamente exclusivos 5 . Com base em estudos anteriores (e.g., Pinto, 2006; Raspa et al., 2001), alguns dos níveis de envolvimento foram combinados, de acordo com critérios de carácter conceptual, resultando em cinco níveis de envolvimento da criança: envolvimento sofisticado (soma de persistente, simbólico, codificado e construtivo), envolvimento diferenciado, atenção focalizada, envolvimento não sofisticado (soma de envolvimento indiferenciado e atenção ocasional) e não envolvido (ver Quadro 1). O acordo interobservador foi calculado para $25 \%$ dos dados recolhidos, tendo variado entre 91,59\% e 99,53\% (Kappa entre 0,57 e 0,80$)$.

Estatuto Desenvolvimental. Foi calculada a idade desenvolvimental a partir das cotações nas cinco subescalas das Griffiths Mental Development

\footnotetext{
${ }^{4}$ Designaremos por educador o responsável pela sala (educador de infância ou auxiliar de acção educativa).

${ }^{5}$ Somente os níveis de envolvimento são examinados neste estudo.
} 
Scales (Griffiths, 1984; versão para investigação de Castro \& Gomes, 1996). O coeficiente Alpha de Cronbach para os dados da escala total, neste estudo, foi de 0,98 e para as sub-escalas variou entre 0,84 e 0,94 .

Comportamento Adaptativo. Usaram-se as Escalas de Comportamento Adaptativo de Vineland (Sparrow, Balla, \& Cicchetti, 1984) que avaliam Comunicação, Actividades da Vida Diária (AVD), Socialização e Motricidade. Neste estudo, foi utilizado o questionário preenchido com base numa entrevista às mães para os domínios Socialização $(\alpha=0,73)$, Comunicação e $\operatorname{AVD}(\alpha=0,93)$.

Temperamento. Os educadores preencheram a Escala da Personalidade da Criança (EPC), traduzida da Childhood Personality Scale (Dibble \& Cohen, 1974) para cada criança. Foram utilizadas quatro dimensões encontradas através de uma análise de componentes principais (Pinto, 2006): Sociabilidade/Adaptabilidade - elevada expressividade verbal, acentuado humor positivo, aquiescência e cooperação com os adultos e reacção positiva da criança à novidade $(\alpha=0,89)$; Atenção - capacidade para se envolver de forma prolongada nas actividades em curso $(\alpha=0,89)$; Controlo socioemocional - auto-regulação relacionada com a competência social, incluindo uma forte componente de controlo motor $(\alpha=0,81)$; Aproximação/Retraimento - empenhamento, entusiasmo e jovialidade relativamente a pessoas e situações $(\alpha=0,79)$.

Qualidade global das salas. Foi utilizada a tradução portuguesa de Pinto e Grego (1994) da Infant/Toddler Environment Rating Scale (ITERS; Harms, Cryer, \& Clifford, 1990) que fornece uma avaliação da qualidade global dos cuidados prestados a crianças até aos 30 meses de idade e é considerada uma medida da qualidade de processo $(\alpha=0,80$ para nota global da escala). ${ }^{6}$

Comportamentos interactivos dos educadores. Foi utilizada a Teaching Styles Rating Scale (TSRS; McWilliam, Scarborough, Bagby, \& Sweeney, 1998), traduzida pela equipa do projecto, que avalia a qualidade de duas dimensões interactivas: comportamentos de ensino (redirecciona, introduz, elabora, segue, informa, reconhece e elogia) e características afectivas (e.g., nível de actividade, responsividade, directividade, consistência das interacções e o tom). Com base nos resultados de uma análise de clusters (Pinto, 2006), os comportamentos de interacção dos educadores foram combinados para formar três variáveis: o comportamento redirecciona, a dimensão de comportamentos directivos-elaborativos (6 comportamentos sem redirecciona) $(\alpha=0,73)$ e a dimensão de afecto $(\alpha=0,92)$.

\footnotetext{
${ }^{6}$ Devido à baixa consistência interna dos dados das subescalas, foi apenas considerada a nota global da escala.
} 
Qualidade do ambiente familiar. Foi aplicada a versão 0-3 da Home Observation for Measurement of the Environment (HOME; Caldwell \& Bradley, 1984), que avalia a qualidade da estimulação no ambiente familiar, através da observação da interacção pais-criança e de uma entrevista acerca dos objectos, acontecimentos e transacções. É composta pelas sub-escalas: Responsividade Emocional e Verbal da Mãe; Aceitação do Comportamento da Criança; Organização do Ambiente Físico e Temporal; Fornecimento de Materiais Lúdicos; Envolvimento Materno com a Criança; Oportunidades para a Variedade na Estimulação Diária. Neste estudo usámos a nota global $(\alpha=0,90)$.

Análises: Foram realizadas análises descritivas para todas as variáveis e análises com base em modelos lineares hierárquicos (Hierarchical Linear Models - HLM; Bryk \& Raudenbush, 1992), no sentido de relacionar a qualidade dos contextos (casa e creche) com os resultados de envolvimento e desenvolvimento das crianças. Utilizámos o HLM, dada a estrutura multi-nível dos dados. Como todas as variáveis nas análises são contínuas centradas na média, a magnitude do efeito para o efeito principal, para as variáveis de níveis 1 e 2, foi calculada através da equação: $\mathrm{B}_{\mathrm{X}} \mathrm{DP}_{\mathrm{x}} / \mathrm{DP}_{\mathrm{Y}}$ (Gutman, Sameroff, \& Cole, 2003) cujo significado prático foi interpretado de acordo com as convenções: 0.10 como um efeito pequeno, 0.30 como um efeito moderado e 0.50 como um efeito importante (NICHD ECCRN \& Duncan, 2003). Sendo os valores $p$ altamente influenciados pelo tamanho da amostra, o significado dos resultados foi interpretado em termos do tamanho do efeito e não apenas com base na significância estatística (Thompson \& Snyder, 1998).

Efeitos no envolvimento: Os efeitos principais da qualidade global das salas e dos comportamentos interactivos dos educadores no envolvimento foram examinados através de análises de regressão hierárquica. Sendo os níveis de envolvimento mutuamente exclusivos, foram criados cinco modelos separados - um para cada nível. Foi efectuada uma análise para cada característica do contexto, que incluiu a idade desenvolvimental, a educação materna $^{7}$, as quatro variáveis de temperamento da criança e uma característica da sala. As variáveis da criança entraram como preditores de efeito fixo de nível 1 e as características da sala entraram como variáveis de efeito fixo de nível 2.

\footnotetext{
${ }^{7}$ Não existindo em Portugal uma medida fidedigna do estatuto socioeconómico (ESE) como o índice de Hollingshead, o nível educativo das mães foi considerado como um indicador do ESE da criança. Esta decisão baseou-se em resultados de estudos prévios que sugerem que a educação materna fornece um índice mais fiel do ESE da família do que o rendimento familiar ou o estatuto profissional (Roberts, Bornstein, Slater, \& Barrett, 1999).
} 
Efeitos no desenvolvimento: Estas análises incluíram os resultados globais da HOME e da ITERS, como variáveis independentes, e os resultados obtidos pelas crianças nas Escalas de Griffiths (Quocientes de desenvolvimento nas sub-escalas Audição e fala, Pessoal-social e Realização) e Vineland (domínios de Comunicação e de Socialização), como variáveis dependentes.

\section{Resultados}

As estatísticas descritivas para as variáveis em estudo são apresentadas no Quadro 2. Estes resultados revelam a existência de heterogeneidade a nível da qualidade do ambiente familiar. Como se pode verificar, a qualidade global das salas de creche é homogeneamente baixa.

\section{Efeitos no envolvimento}

Qualidade da sala. Quando são consideradas a escolaridade materna e as características de desenvolvimento e de temperamento da criança, a qualidade não está significativamente associada ao tempo que as crianças passam em envolvimento sofisticado, em envolvimento diferenciado e em atenção focalizada (Quadro 3). Relativamente ao envolvimento não sofisticado, embora a associação não seja estatisticamente significativa, a qualidade da sala tem um efeito modesto, indicando que, em salas com qualidade mais baixa, as crianças têm mais probabilidade de serem observadas em comportamentos de envolvimento não sofisticado e têm tendência para passar mais tempo não envolvidas. Relativamente às variáveis da criança, a educação materna não contribui para o modelo hierárquico considerado. A idade desenvolvimental e as características de temperamento contribuem para a percentagem de tempo que as crianças passam nos diferentes níveis de envolvimento. 
Quadro 2. Resultados descritivos

\begin{tabular}{|c|c|c|c|c|}
\hline & Escala & Min.-Max. & $\mathbf{M}$ & DP \\
\hline Qualidade global das salas de creche - ITERS & $1-7$ & $1.76-3.47$ & 2.55 & 0.42 \\
\hline Qualidade do contexto familiar - HOME & $0-45$ & $11.00-45.00$ & 35.21 & 7.59 \\
\hline \multicolumn{5}{|l|}{ Envolvimento observado - SAQE III } \\
\hline Envolvimento sofisticado & $\begin{array}{c}\% \text { intervalos } \\
\text { de tempo }\end{array}$ & $0.00-38.84$ & 8.07 & 8.30 \\
\hline Envolvimento diferenciado & " & $26.79-71.25$ & 45.74 & 9.13 \\
\hline Atenção focalizada & $"$ & $10.26-40.71$ & 21.61 & 6.58 \\
\hline Envolvimento não sofisticado & $"$ & $1.47-25.32$ & 10.60 & 4.54 \\
\hline Não envolvido & $"$ & $2.92-35.42$ & 14.01 & 6.60 \\
\hline \multicolumn{5}{|l|}{ Estatuto desenvolvimental - GRIFFITHS } \\
\hline Idade desenvolvimental (meses) & & $13.50-47.67$ & 27.30 & 8.23 \\
\hline Quociente de desenvolvimento & & $79.45-132.35$ & 103.56 & 9.87 \\
\hline Realização (QD) & & $70.97-141.67$ & 109.83 & 14.69 \\
\hline Audição e fala (QD) & & $63.15-145.45$ & 99.65 & 15.02 \\
\hline Pessoal-social (QD) & & 64.71-141.18 & 102.35 & 13.41 \\
\hline \multicolumn{5}{|l|}{ Comportamento adaptativo - VINELAND } \\
\hline Total & & $56.00-186.00$ & 121.28 & 29.96 \\
\hline Comunicação & & $19.00-82.00$ & 45.16 & 14.05 \\
\hline Socialização & & $28.00-53.00$ & 41.17 & 4.76 \\
\hline \multicolumn{5}{|l|}{ Temperamento - EPC } \\
\hline Sociabilidade/Adaptabilidade & $0-6$ pontos & $2.19-5.81$ & 4.12 & 0.87 \\
\hline Atenção & $"$ & $0.75-5.75$ & 3.65 & 1.05 \\
\hline Controlo socioemocional & $"$ & $1.50-5.80$ & 3.70 & 0.95 \\
\hline Aproximação/Retraimento & $"$ & $2.09-6.00$ & 4.54 & 0.80 \\
\hline \multicolumn{5}{|l|}{ Comportamentos de ensino - TSRS } \\
\hline Redirecciona & 1-7 pontos & $2.75-6.50$ & 4.81 & 0.79 \\
\hline Directivos-elaborativos (s/ redirecciona) & $"$ & $2.46-4.33$ & 3.39 & 0.50 \\
\hline Média Afecto & $1-5$ pontos & $2.36-4.57$ & 3.57 & 0.54 \\
\hline
\end{tabular}

Redireccionamentos. Não existem associações entre a utilização de redireccionamentos pelo educador e os níveis de envolvimento (Pinto, 2006), pelo que estes resultados não serão apresentados.

Comportamentos de ensino directivos-elaborativos. A utilização de comportamentos de ensino directivos-elaborativos pelas educadoras está significativamente relacionada com o envolvimento diferenciado e com o não envolvimento (Quadro 4). Em salas nas quais os educadores utilizam mais frequentemente comportamentos directivos-elaborativos, sendo responsivos e contingentes, as crianças passam mais tempo a interagir de forma activa e menos tempo não envolvidas. A dimensão de comportamentos directivos-elaborativos não está significativamente associada com os restantes três níveis de envolvimento. 
Quadro 3. Relação entre qualidade global da sala, características de temperamento e envolvimento da criança em contexto de creche

\begin{tabular}{|c|c|c|c|c|c|c|c|c|c|c|c|c|c|c|c|}
\hline & \multicolumn{3}{|c|}{$\begin{array}{l}\text { RQ }^{9} \text { Envolvimento } \\
\text { sofisticado }\end{array}$} & \multicolumn{3}{|c|}{$\begin{array}{c}\text { Envolvimento } \\
\text { diferenciado }\end{array}$} & \multicolumn{3}{|c|}{ Atenção focalizada } & \multicolumn{3}{|c|}{$\begin{array}{c}\text { Envolvimento } \\
\text { não sofisticado }\end{array}$} & \multicolumn{3}{|c|}{ Não envolvido } \\
\hline & $B$ & $S E$ & $M E^{10}$ & $B$ & $S E$ & $M E$ & $B$ & $S E$ & $M E$ & $B$ & $S E$ & $M E$ & $B$ & $S E$ & $M E$ \\
\hline Intercept & $2.53^{* * *}$ & 0.11 & & $45.03^{* * *}$ & 0.81 & & $21.61^{* * *}$ & 0.53 & & $10.60^{* * *}$ & 0.50 & & $14.01 * * *$ & 0.62 & \\
\hline \multicolumn{16}{|l|}{ Efeitos Principais } \\
\hline Qualidade Global da Sala de Creche & 0.08 & 0.24 & - & $4.57^{*}$ & 1.88 & & -0.51 & 1.23 & -0.03 & -1.03 & 1.53 & -0.10 & $-4.26^{* *}$ & 1.09 & -0.29 \\
\hline Educação da mãe (anos) & -0.01 & 0.02 & -0.03 & -0.09 & 0.18 & -0.04 & -0.02 & 0.11 & -0.01 & $0.18^{*}$ & 0.08 & 0.18 & -0.02 & 0.08 & -0.01 \\
\hline Idade desenvolvimental (meses) & $0.11 * * *$ & 0.01 & 0.63 & $-0.21 *$ & 0.10 & -0.19 & -0.14 & 0.08 & -0.18 & -0.02 & 0.06 & -0.04 & $-0.34 * * *$ & 0.07 & -0.42 \\
\hline Sociabilidade /Adaptabilidade & $0.48^{* * *}$ & 0.13 & 0.29 & 1.76 & 1.15 & 0.17 & $-3.01 * *$ & 0.90 & -0.40 & $-1.88^{* *}$ & 0.55 & -0.36 & 1.13 & 1.01 & 0.15 \\
\hline Atenção & -0.00 & 0.09 & - & -1.21 & 1.01 & -0.14 & 0.71 & 0.66 & 0.11 & $1.32 * *$ & 0.43 & 0.31 & -0.76 & 0.57 & -0.12 \\
\hline Controlo socioemocional & -0.07 & 0.05 & -0.05 & $-1.63^{*}$ & 0.81 & -0.17 & $1.67^{* *}$ & 0.60 & 0.24 & $0.89^{*}$ & 0.35 & 0.19 & -0.84 & 0.49 & -0.12 \\
\hline Aproximação/Retraimeno & $-0.22 *$ & 0.10 & - & 0.29 & 1.07 & .- & $3.01 * * *$ & 0.81 & 0.37 & -0.84 & 0.50 & -0.15 & -0.55 & 0.67 & -0.07 \\
\hline
\end{tabular}

Nota: $* p<0,05 ; * * p<0,01 ; * * * p<0,001$

Quadro 4. Relação entre comportamentos directivos-elaborativos, características de temperamento e envolvimento da criança em contexto de creche

\begin{tabular}{|c|c|c|c|c|c|c|c|c|c|c|c|c|c|c|c|}
\hline & \multicolumn{3}{|c|}{$\begin{array}{c}\text { RQ Envolvimento } \\
\text { sofisticado }\end{array}$} & \multicolumn{3}{|c|}{$\begin{array}{c}\text { Envolvimento } \\
\text { diferenciado }\end{array}$} & \multicolumn{3}{|c|}{ Atenção focalizada } & \multicolumn{3}{|c|}{$\begin{array}{c}\text { Envolvimento não } \\
\text { sofisticado }\end{array}$} & \multicolumn{3}{|c|}{ Não envolvido } \\
\hline & $B$ & $S E$ & $M E$ & $B$ & $S E$ & $M E$ & $B$ & $S E$ & $M E$ & $B$ & $S E$ & $M E$ & $B$ & $S E$ & $M E$ \\
\hline Intercept & $2.46^{* * *}$ & 0.11 & & $45.74 * * *$ & 0.87 & & $21.61^{* * *}$ & 0.53 & & $10.60^{* * *}$ & 0.50 & & $14.01 * * *$ & 0.65 & \\
\hline \multicolumn{16}{|l|}{ Efeitos Principais } \\
\hline Comportamentos de ensino & 0.03 & 0.24 & 0.01 & $3.41^{*}$ & 1.63 & 0.19 & -0.16 & 1.21 & -0.01 & -0.71 & 0.9 & -0.08 & $-2.87 *$ & 1.24 & -0.22 \\
\hline Educação da mãe (anos) & -0.01 & 0.02 & -0.03 & -0.09 & 0.19 & -0.04 & -0.02 & 0.11 & -0.01 & $0.18^{*}$ & 0.08 & 0.18 & 0.01 & 0.08 & 0.01 \\
\hline Idade desenvolvimental (meses) & $0.11^{* * *}$ & 0.01 & 0.63 & $-0.23^{*}$ & 0.10 & -0.21 & -0.14 & 0.08 & -0.18 & -0.02 & 0.06 & -0.04 & $-0.34^{* * *}$ & 0.08 & -0.42 \\
\hline Controlo socioemocional & -0.04 & 0.07 & -0.03 & $-1.75^{*}$ & 0.82 & -0.18 & $1.69^{* *}$ & 0.60 & 0.24 & $0.90^{* *}$ & 0.34 & 0.19 & -0.75 & 0.48 & -0.11 \\
\hline Aproximação/Retraimento & -0.12 & 0.10 & -0.07 & -1.57 & 1.32 & -0.14 & $3.07 * * *$ & 0.81 & 0.37 & -0.73 & 0.56 & -0.13 & -0.07 & 0.65 & -0.01 \\
\hline
\end{tabular}

Nota: $* p<0,05 ; * * p<0,01 ; * * * p<0,001$

\footnotetext{
${ }^{9}$ Raiz Quadrada

${ }^{10}$ Magnitude do efeito
} 
Características afectivas. Relativamente à dimensão de afecto do educador encontrou-se uma associação de pequena magnitude com o envolvimento diferenciado e com o envolvimento não sofisticado (Quadro 5). Estes dados indicam uma tendência para que, em salas com educadores mais calorosos, as crianças tenham mais probabilidade de se envolverem no nível diferenciado (interagindo activamente com adultos, pares e objectos) e menos probabilidade de se envolverem em comportamentos repetitivos ou de passarem o tempo simplesmente a monitorizar o ambiente nas salas.

\section{Efeitos no desenvolvimento}

Qualidade da sala. Um aumento na qualidade das salas não se encontra associado, de forma estatisticamente significativa, a resultados mais elevados na Griffiths, constatando-se um efeito de pequena magnitude relativamente à subescala de Realização. No que diz respeito aos domínios de comportamento adaptativo (Comunicação e Socialização) verificam-se, igualmente, efeitos de pequena magnitude, embora as associações não sejam estatisticamente significativas (Quadro 6).

Qualidade do ambiente familiar. Uma qualidade mais elevada a este nível encontra-se associada, de forma estatisticamente significativa, a resultados mais elevados na Griffiths, embora a magnitude do efeito seja pequena. Quando os resultados na HOME são mais elevados, as crianças obtêm resultados mais elevados nas subescalas Audição e fala, Pessoal-social e Realização. Um aumento na qualidade do ambiente familiar encontra-se associado, de forma estatisticamente significativa, a um aumento nos resultados obtidos na Vineland (Comunicação e Socialização), apesar da magnitude dos efeitos ser pequena (Quadro 6). 
Quadro 5. Relação entre afecto, características de temperamento e envolvimento da criança em contexto de creche

\begin{tabular}{|c|c|c|c|c|c|c|c|c|c|c|c|c|c|c|c|}
\hline & \multicolumn{3}{|c|}{$\begin{array}{c}\text { RQ Envolvimento } \\
\text { sofisticado }\end{array}$} & \multicolumn{3}{|c|}{$\begin{array}{c}\text { Envolvimento } \\
\text { diferenciado }\end{array}$} & \multicolumn{3}{|c|}{ Atenção focalizada } & \multicolumn{3}{|c|}{$\begin{array}{l}\text { Envolvimento } \\
\text { não sofisticado }\end{array}$} & \multicolumn{3}{|c|}{ Não envolvido } \\
\hline & $B$ & $S E$ & $M E$ & $B$ & $S E$ & $M E$ & $B$ & $S E$ & $M E$ & $B$ & $S E$ & $M E$ & $B$ & $S E$ & $M E$ \\
\hline Intercept & $2.46^{* * *}$ & 0.11 & & $45.74 * * *$ & 0.89 & & $21.61^{* * *}$ & 0.53 & & $10.60^{* * *}$ & 0.49 & & $14.01 * * *$ & 0.67 & \\
\hline \multicolumn{16}{|l|}{ Efeitos Principais } \\
\hline Afecto & -0.15 & 0.19 & -0.06 & 2.74 & 1.71 & 0.16 & -0.37 & 1.02 & -0.03 & -1.26 & 0.73 & -0.15 & -0.83 & 1.20 & -0.07 \\
\hline Educação da mãe (anos) & -0.01 & 0.02 & -0.03 & -0.10 & 0.19 & -0.05 & -0.02 & 0.11 & -0.01 & $0.18 *$ & 0.08 & 0.18 & 0.03 & 0.08 & 0.02 \\
\hline $\begin{array}{l}\text { Idade desenvolvimental (meses) } \\
\text { Sociabilidade }\end{array}$ & \multicolumn{14}{|c|}{ Sociabilidade } & -0.45 \\
\hline /Adaptabilidade & $0.43 * *$ & 0.13 & 0.26 & 2.23 & 1.17 & 0.21 & $-3.06^{* *}$ & 0.87 & -0.40 & $-1.98^{* *}$ & 0.57 & -0.38 & 1.08 & 1.11 & 0.14 \\
\hline Atenção & -0.03 & 0.13 & -0.02 & -0.97 & 1.13 & -0.11 & 0.71 & 0.59 & 0.11 & $1.36^{* *}$ & 0.40 & 0.31 & -1.03 & 0.65 & -0.16 \\
\hline Controlo sócio-emocional & -0.04 & 0.07 & -0.03 & $-1.66^{*}$ & 0.83 & -0.17 & $1.67 * *$ & 0.59 & 0.24 & $0.89^{*}$ & 0.35 & 0.19 & -0.75 & 0.51 & -0.11 \\
\hline Aproximação/Retraimento & -0.11 & 0.10 & -0.06 & -1.47 & 1.32 & -0.13 & $3.09^{* * *}$ & 0.81 & 0.38 & -0.69 & 0.55 & -0.12 & -0.32 & 0.66 & -0.04 \\
\hline
\end{tabular}

Nota: $* p<0,05 ; * * p<0,01 ; * * * \mathrm{p}<0,001$ 
Quadro 6. Relação entre qualidade dos contextos e os resultados obtidos na Griffiths e na Vineland

\begin{tabular}{|c|c|c|c|c|c|c|c|c|c|c|c|c|c|c|c|}
\hline & \multicolumn{9}{|c|}{ Griffiths } & \multicolumn{6}{|c|}{ Vineland } \\
\hline & \multicolumn{3}{|c|}{ Audição e fala } & \multicolumn{3}{|c|}{ Pessoal-social } & \multicolumn{3}{|c|}{ Realização } & \multicolumn{3}{|c|}{ Comunicação } & \multicolumn{3}{|c|}{ Socialização } \\
\hline & $B$ & $S E$ & $M E$ & $B$ & $S E$ & $M E$ & $B$ & $S E$ & $M E$ & $B$ & $S E$ & $M E$ & $B$ & $S E$ & $M E$ \\
\hline Intercept & 99.65 & 1.44 & & 102.4 & 1.59 & & 109.8 & 1.76 & & 45.16 & 2.04 & & 41.17 & 0.60 & \\
\hline ITERS & 3.01 & 2.84 & 0.08 & 2.05 & 3.98 & 0.06 & 5.52 & 3.45 & 0.16 & 8.43 & 4.24 & 0.25 & 2.38 & 1.50 & 0.21 \\
\hline HOME & $0.72 * * *$ & 0.16 & 0.36 & $0.43 * *$ & 0.14 & 0.25 & $0.46^{*}$ & 0.20 & 0.24 & $0.36 * *$ & 0.10 & 0.19 & $0.13 * *$ & 0.04 & 0.21 \\
\hline
\end{tabular}

Nota: $* p<0,05 ; * * p<0,01 ; * * * p<0,001$

\section{Discussão}

Este estudo contribuiu para esclarecer a relação entre as caracteristicas da criança, a qualidade dos contextos familiar e de creche, as interacções diárias da criança com o meio, isto é, o seu envolvimento, e o desenvolvimento. Tal como em estudos anteriores (e.g., de Kruif \& McWilliam, 1999), verificou-se que a idade desenvolvimental e as características de temperamento contribuem para a percentagem de tempo que as crianças passam em diferentes níveis de envolvimento.

Tendo presente que a maioria das crianças estava em salas de creche com qualidade inadequada, de acordo com os critérios de Harms et al., (1990), e que a baixa variabilidade das cotações de qualidade na amostra deste estudo reduziram o poder das análises para detectar associações entre qualidade dos contextos e resultados das crianças, acreditamos que mesmo a modesta magnitude dos efeitos encontrados fornece evidência razoável de associações com significado entre a qualidade dos contextos e das interacções educativas e o envolvimento e desenvolvimento das crianças. Consideramos que a nota global de qualidade utilizada, ao incluir aspectos de estrutura e de processo, não possibilitou uma análise mais fina destas associações, a qual seria possível através da utilização de dados fiáveis das subescalas. Adicionalmente, como não existiam salas avaliadas com um nível de qualidade global considerado bom ou excelente, os dados limitaram-se a evidenciar os efeitos de níveis de qualidade inadequada ou minimamente adequada no envolvimento e no desenvolvimento. Assim, mesmo controlando o efeito de características da criança, foi evidente uma associação entre níveis mais adequados de qualidade global das salas e uma menor frequência de comportamentos de não envolvimento. Verificou-se igualmente uma tendência para 
que em salas de qualidade mais elevada, as crianças passem menos tempo em comportamentos repetitivos, de envolvimento não sofisticado. Tem sido relatada uma relação positiva entre a qualidade geral do ambiente educativo e processos mais próximos relativamente a essa qualidade, nomeadamente o envolvimento das crianças nas tarefas (Raspa et al., 2001; de Kruif et al., 2001). Os autores concluíram que crianças, em salas com qualidade mais elevada, demonstram jogo e actividades de nível mais complexo com pares, objectos e adultos (e.g., Raspa et al., 2001), enquanto em salas de qualidade mais baixa, as crianças têm tendência para estar ocupadas em níveis mais baixos, tais como jogo solitário, comportamentos repetitivos ou não envolvimento. Raspa et al., (2001) referem que a qualidade global da sala (medida pela ITERS) se encontra positivamente associada com o tempo que as crianças passam em comportamentos de nível sofisticado e negativamente associada com o tempo em envolvimento não sofisticado (isto é, comportamentos repetitivos ou atenção ocasional).

Os resultados deste estudo evidenciaram, ainda, efeitos positivos da qualidade das salas na linguagem, na socialização e na cognição, mesmo quando se controlaram os efeitos da qualidade do ambiente familiar. A qualidade dos serviços de educação de infância, medida pela ITERS e por outros instrumentos dos mesmos autores, tem sido consistentemente associada ao desenvolvimento cognitivo e às competências sociais das crianças (Burchinal, Roberts, Hooper, \& Zeisel, 2000), mesmo quando as características do meio familiar são controladas (e.g., Burchinal, Roberts, Nabors, \& Bryant, 1996). Parecem-nos, pois, relevantes estes efeitos da qualidade, especialmente no contexto português, onde os resultados de um estudo recente revelam uma qualidade global entre inadequada e mínima em 160 salas de creche avaliadas com a ITERS (Barros, 2007). De acordo com a teoria desenvolvimental, o desenvolvimento é fortemente influenciado por relações interpessoais proximais (Bronfenbrenner \& Morris, 1998). Com efeito, no presente estudo, em salas com educadoras que utilizavam mais comportamentos directivos-elaborativos, sendo responsivas e contingentes, as crianças tinham tendência para passar menos tempo não envolvidas e mais tempo em comportamentos de envolvimento diferenciado (i.e., interagiam activamente com adultos, pares e objectos). Estudos prévios apontam também para a necessidade de os educadores combinarem comportamentos responsivos e directivos para que a sua interacção possa ter um efeito positivo no envolvimento das crianças (McWilliam, Scarborough, \& Kim 2003) e concluem que os que utilizam de forma equilibrada comportamentos de interacção responsivos e directivos obtêm maior sucesso na promoção de níveis sofisticados de envolvimento (de Kruif, Zully, McWilliam, Scarborough, \& Sloper, 1998).

Verificou-se ainda que, com educadoras mais afectuosas, as crianças tinham mais probabilidade de se envolverem no nível diferenciado e menos 
probabilidade de se envolverem num nível não sofisticado, isto é, de interagir com comportamentos repetitivos ou simplesmente de monitorizar o ambiente, o que era esperado e vai no sentido de resultados de estudos prévios (Ridley, McWilliam, \& Oates., 2000; Raspa et al., 2001). Estes resultados parecem indicar que a utilização de comportamentos directivos-elaborativos e de interacções afectuosas por parte das educadoras de infância e das auxiliares de acção educativa têm efeitos positivos na forma como as crianças interagem com o meio.

Os resultados obtidos demonstram a necessidade de se melhorar a qualidade dos cuidados prestados a crianças mais novas através de esforços sistemáticos no sentido de melhorar o processo educativo a nível de aspectos estruturais e de características interactivas dos prestadores de cuidados. Será necessário repensar as políticas, estratégias de financiamento e práticas de creditação ou credenciação que regulamentam os serviços nacionais de prestação de cuidados à infância. Estas mudanças dependem de opções governamentais ao nível da legislação, apoio à investigação e divulgação dos seus resultados, bem como à criação e apoio de programas nacionais (Aguiar, Bairrão, \& Barros, 2002).

\section{Referências}

Aguiar, C., Bairrão, J., \& Barros, S. (2002). Contributos para o estudo da qualidade em contexto de creche na Área Metropolitana do Porto. Infância e Educação: Investigação e Práticas, 5, 7-28.

Barros, S. (2007). Qualidade em contexto de creche: ideias e práticas. Dissertação de Doutoramento não publicada, Universidade do Porto, Porto.

Bronfenbrenner U., \& Morris, P. A. (1998). The ecology of developmental process. In W. Damon (Series Ed.), \& R. M. Lerner (Vol. Ed.), Handbook of Child Psychology: Vol. 1 Theoretical Models of Human Development. New York: Wiley.

Bryk, A. S., \& Raudenbush, S. W. (1992). Hierarchical linear models: Applications and data analysis methods. Newbury Park, CA: Sage.

Burchinal. M. R., Roberts, J. E., Hooper, S., \& Zeisel, S. A. (2000). Cumulative risk and early cognitive development: A comparison of statistical risk models. Developmental Psychology, 36, 793-807.

Burchinal. M. R., Roberts, J. E., Nabors, L. A., \& Bryant, D. M. (1996). Quality of center child care and infant cognitive and language development. Child Development, 67, 606-620.

Caldwell, B., \& Bradley, R. (1984). Home Observation for the Measurement of the Environment. Little Rock: University of Arkansas at Little Rock.

Campbell, F. A., Wasik, B. H., Pungello, E., Burchinal M., Barbarin, O., Kainz, K., et al. (2008). Young adult outcomes of the Abecedarian and CARE early childhood educational interventions. Early Childhood Research Quarterly, 23, 452466. 
de Kruif, R. E. L., \& McWilliam, R. A. (1999). Multivariate relationships among development age, global engagement and observed child engagement. Early Childhood Researche Quarterly, 14, 515-536.

de Kruif, R. E. L., Zully, R. A., McWilliam, R. A., Scarborough, A. A., \& Sloper, K. M. (1998). Cases of responsiveness and directiveness and different levels of teaching. University of North Carolina at Chapel Hill. Manuscrito não publicado.

Dibble, E., \& Cohen, D. J. (2000). Escala de Personalidade da Criança - EPC. (Equipa do projecto A qualidade das interacções da criança em contexto familiar e de creche e o seu impacto no desenvolvimento sociocognitivo da crian$c ̧ a$ ). (Centro de Psicologia da Universidade do Porto, FPCEUP). (Obra original publicada em 1974).

Griffiths, R. (1996). As Escalas Griffiths adaptadas ao Português: Versão parcial para investigação. (Castro, S. L., \& Gomes, I.). (Laboratório de Fala, Faculdade de Psicologia e de Ciências da Educação da Universidade do Porto). (Obra original publicada em 1984).

Harms, T., Cryer, D., \& Clifford, R. M. (1996). Escala de Avaliação do Ambiente de Creche - EAAC. (Pinto, A. I., \& Grego, T.). (Obra original publicada em 1990).

McWilliam, R. A., \& Bailey, D. B. (1995). Effects of classroom social structure and disability on engagement. Topics in Early Childhood Special Education, 15, 123-147.

McWilliam, R., \& de Kruif, R. (2000). O Sistema de Avaliação da Qualidade do Envolvimento III - SAQE III.. (Equipa do projecto A qualidade das interacções da criança em contexto familiar e de creche e o seu impacto no desenvolvimento sociocognitivo da criança). (Centro de Psicologia da Universidade do Porto, FPCEUP). (Obra original publicada em 1998).

McWilliam, R., Scarborough, A., Bagby, J., \& Sweeney, A. (2000). A Escala de Avaliação dos Estilos de Ensino - EAEE. (Equipa do projecto A qualidade das interacções da criança em contexto familiar e de creche e o seu impacto no desenvolvimento sociocognitivo da criança). (Centro de Psicologia da Universidade do Porto, FPCEUP). (Obra original publicada em 1996).

McWilliam, R. A., Scarborough, A. A., \& Kim, H. (2003). Adult interactions and child engagement. Early Education and Development, 14, 7-27.

Gutman, L. M., Sameroff, A. J., \& Cole, R. (2003). Academic growth curve trajectories from $1^{\text {st }}$ grade to $12^{\text {th }}$ grade: Effects of multiple social risk factors and preschool child factors. Developmental Psychology, 39, 777-790.

NICHD Early Child Care Research Network \& Duncan, G. J. (2003). Modelling the impact of child care quality on children's preschool cognitive development. Child Development, 74, 1454-1475.

NICHD Early Child Care Research Network (2006). Child care effect sizes for the NICHD study of early child care and youth development. American Psychologist, 2, 99-116.

Pinto, A. I. (2006). O envolvimento da criança em contexto de creche: os efeitos de características da criança, da qualidade do contexto e das interacções educa- 
tivas. Faculdade de Psicologia e de Ciências da Educação da Universidade do Porto. Dissertação de doutoramento não publicada.

Pinto, A. I., Aguiar, C., Barros, S., Pessanha, M., \& Bairrão, J. (2006). Relações entre idade desenvolvimental, dimensões do comportamento adaptativo e envolvimento observado. Análise Psicológica, 4 (XXIV), 447-466.

Raspa, M. J., McWilliam, R. A., \& Ridley, S. M. (2001). Child care quality and children's engagement. Early Education and Development, 12, 209-224.

Ridley, S. M., McWilliam, R. A., \& Oates, C. S. (2000). Observed engagement as an indicator of child care program quality. Early Education \& Development, 11, 133-146.

Roberts, E., Bornstein, M. H., Slater, A. M., \& Barrett, J. (1999). Early cognitive development and parental education. Infant and Child Development, 8, 49-62.

Sameroff, A., \& Fiese, B. (2000). Transactional regulation and early intervention. In J. P. Shonkoff \& S. J. Meisels (Eds.), Handbook of Early Childhood Intervention. Cambridge: Cambridge University Press.

Shonkoff, J., \& Phillips, D. (2000). From neurons to neighbourhoods: The science of early childhood development. Washington, DC: National Academy Press.

Sparrow, S. S., Balla, D. A., \& Cicchetti, D. V. (1984). Vineland Adaptive Behaviour Scales: Interview Edition Survey Form Manual. Minnesota: American Guidance Service.

Thompson, B., \& Snyder, P. A. (1998). Statistical significance and reliability analysis in recent Journal of Counseling \& Development research articles. Journal of Counselling and Development, 76, 436-441. 